\title{
RESEÑA DEL LIBRO AGROECOLOGÍA: CIENCIA Y POLÍTICA
}

\section{Book Review: Agroecology: Science and Politics}

\section{Sergio Moctezuma Pérez \\ Instituto de Ciencias Agropecuarias y Rurales, Universidad Autónoma del Estado de México, \\ Toluca, Estado de México, México Correo-e: smoctezumap@uaemex.mx}

Cómo citar: Moctezuma Pérez, S. (2019). Reseña del libro Agroecología: ciencia y política. Ciencia, Ambiente y Clima, 2(1), 53-57. doi: https://doi.org/10.22206/cac.2019.v2i1.pp53-57

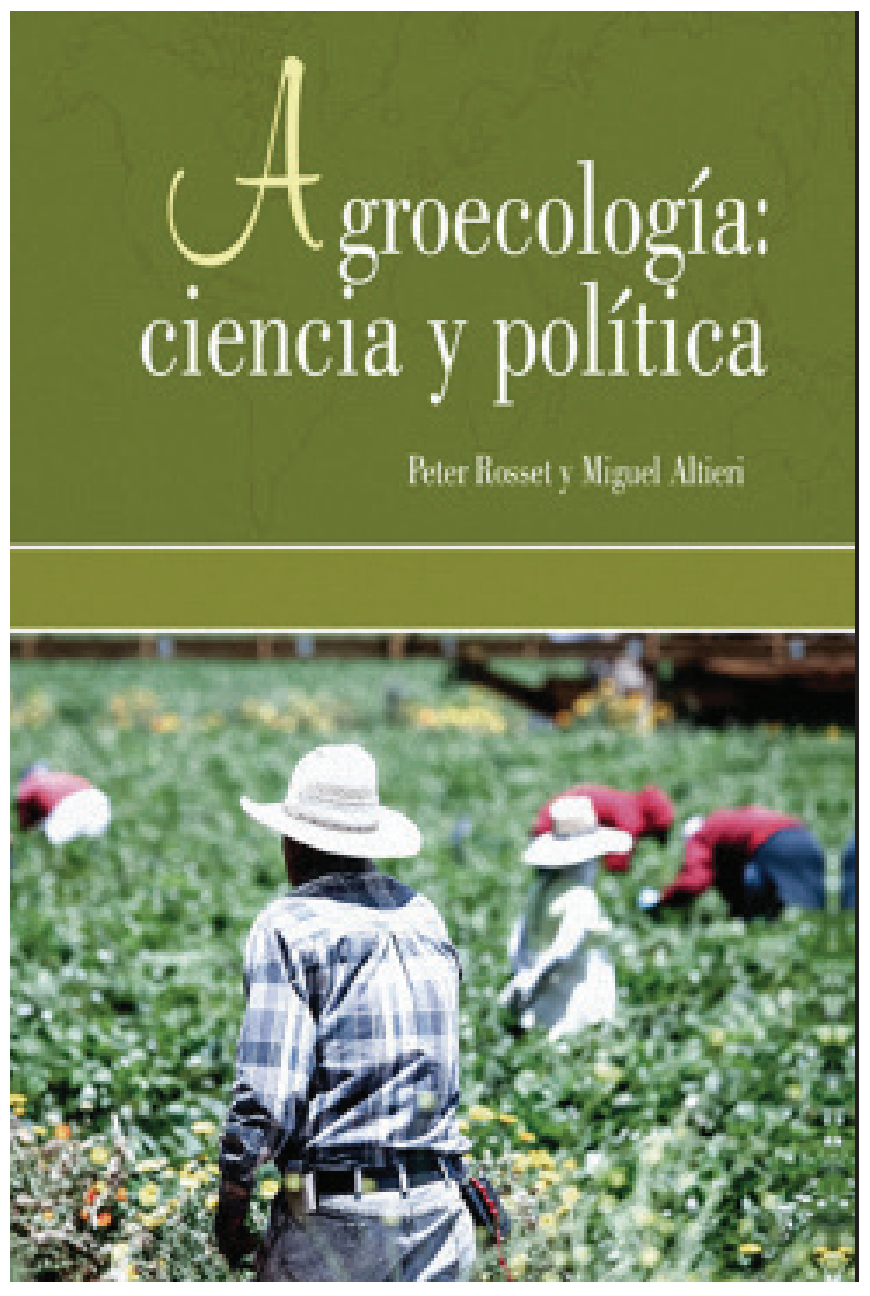

El libro Agroecología: ciencia y política escrito por Peter Rosset y Miguel Altieri fue publicado por vez primera en 2017 en versión inglesa. Ahora, en 2019, la Universidad Autónoma de Zacatecas y la editorial Miguel Ángel Porrúa lo publican en espańol. El libro forma parte de la serie Iniciativas para Estudios Agrarios: Cambios Agrarios y Estudios del Campesinado. Ambos autores son especialistas en el estudio de la relación entre ambiente y los seres humanos. En este caso, centrándose en la agroecología como un tema que involucra alimentación, campesinado, agricultura, movimientos sociales, conocimientos técnico-científicos y conocimientos locales, ecológicos, tradicionales e indígenas.

El libro consta de un breve prefacio, una introducción y cinco capítulos. Son 187 páginas que el lector seguirá fácilmente y en las que se encuentran citados poco más de trescientos trabajos científicos que sustentan el contenido. El tema de la agroecología es conocido entre biólogos, agrónomos, antropólogos, tomadores de decisiones, campesinos e indígenas, así como movimientos y colectivos que pugnan por una agricultura justa, limpia, armónica y demás. El libro, aunque trate temas de ecología 
y de ciencias sociales en general, es de fácil lectura porque los autores han sabido explicar los tecnicismos y los debates que se mencionan.

En la introducción del libro los autores parten del principio de que la agroecología tiene un componente político muy fuerte. Además, señalan que la agroecología es: a) una ciencia que estudia los agroecosistemas; b) un conjunto de prácticas para cultivar sosteniblemente sin el uso de agroquímicos; y c) un movimiento que pugna por una agricultura ecológicamente sostenible y socialmente justa. Estas tres perspectivas de la agroecología han llamado la atención de diversas instituciones educativas, empresas, gobiernos y organizaciones no gubernamentales, sin embargo, las versiones de agroecología que ostentan suelen diferir de la versión original y también entre las actuales.

Estas diferencias se encuentran en el campo técnico y político de la agroecología. Por estas razones, los autores titularon su introducción con el subtítulo: La agroecología en una encrucijada. Para los autores, existe una agroecología liderada por un bando institucional que la concibe como unas herramientas adicionales para la agricultura convencional y la producción de alimentos industrializados. Por otro lado, existe una agroecología propia de un bando de científicos, activistas y militantes, agricultores ecológicos, ONG's y movimientos sociales. Para todos ellos, la agroecología es una alternativa a la producción industrial y una vía para cambiar nuestro actual sistema alimentario.

¿Qué clase de agroecología será la elegida por todos? Esta y varias preguntas más nos hacen los autores para dejar en claro que cuando hablamos de agroecología debemos tener en la mente una idea más o menos homogénea. Pero esa idea no surge de una agricultura convencional y moderna pintada con un poquito de color e ideología verde proveniente de la agroecología. Por el contrario, es una idea sustentada en la agricultura tradicional, principalmente familiar y campesina, que puede ayudar a solucionar algunos problemas medioambientales a los que nos enfrentamos en la actualidad muchas de las sociedades de este planeta.

El primer capítulo del libro lleva por nombre "Principios de la agroecología" y a su vez se divide en seis secciones. Una de las premisas que fundamenta este capítulo es que las raíces de la agroecología se encuentran en la racionalidad de la agricultura llevada a cabo por grupos indígenas y campesinos en muchos lugares del mundo. Esta racionalidad se abreva del conocimiento ecológico tradicional, de los procesos de experimentación, así como del ingenio de las personas que poseen y manejan los sistemas agrícolas tradicionales. La agroecología combina el conocimiento de estos grupos humanos con las ciencias agronómicas y ecológicas.

La segunda premisa del capítulo es que la agroecología no se basa en recetas técnicas, por el contrario, se sustenta en principios. Lo anterior nos permite entender que detrás de la amplia diversidad de agroecosistemas existentes, hay principios que los caracterizan: 1) niveles muy altos de biodiversidad, 2) sistemas para conservar y gestionar los recursos edáficos e hídricos, 3) biodiversidad para la soberanía alimentaria a diversas escalas, 4) resiliencia y robustez para mitigar diversos tipos de riesgos, 5) conocimiento tradicional con constantes innovaciones, y 6) valores culturales y formas de organización social colectivas para acceder a los recursos y beneficios.

Este primer capítulo contiene un breve estado del arte respecto a diversos temas, por ejemplo: la diversidad genética, la diversidad de especies cultivadas, la integración del ganado en sistemas silvopastoriles, el papel ecológico de la biodiversidad en los agroecosistemas. Sobre este último, los autores explican el concepto de diversidad funcional y la redundancia en los sistemas agrícolas: “(...) hay muchas más especies que funciones, por lo que la redundancia es una característica de la biodiversidad y forma parte del agroecosistema (...) los componentes que 
pueden parecer redundantes al final quizá resulten fundamentales ante un cambio medioambiental inesperado" (Rosset y Altieri, 2019: p. 31).

Otros temas que se abordan son la matriz ecológica, los principios para el diseño de sistemas agrícolas diversificados, sobreproducción, regulación de plagas, la diversidad y resiliencia ante el cambio climático, la conservación agroecológica de las fincas, los cambios en la biología de los suelos, la evolución de la productividad, el síndrome de producción y la diversificación intencional. Las temáticas son amplias, pertinentes y bien explicadas mediante ejemplos y referencias académicas clásicas y contemporáneas. Quienes han seguido la trayectoria de estos autores, en sus diversas publicaciones, encontrarán que este capítulo es un concentrado de información que suele hallarse en libros completos.

El segundo capítulo se titula "Historia y corrientes del pensamiento agroecológico". Si los especialistas en ciencias biológicas, ecológicas y agronómicas encontraron en el primer capítulo un gran resumen de los aportes que sus ciencias han hecho a la agroecología, entonces los especialistas en ciencias sociales y teoría social hallarán en el segundo capítulo un breve, pero interesante análisis de temas como la descampesinización y recampesinización del mundo rural. En una primera sección de este capítulo, los autores nos presentan la genealogía de aquellas personas que han contribuido a estudiar, aplicar y difundir la agroecología desde diversas partes del mundo.

Los autores narran los aportes de Rudolf Steiner, científico alemán que impulsó la agricultura biodinámica y los de Sir Albert Howard, pionero de la agricultura orgánica a partir de sus observaciones de los sistemas y prácticas agrícolas en la India. A su vez, se encuentra Franklin Hiram King quien documentó a principios del siglo $\mathrm{xx}$ los sistemas agrícolas tradicionales de China, así como los aportes sobre agricultura orgánica que hizo Lady Eve Balfour. También se menciona a Basil M. Bensin, quien usó por vez primera el concepto de agroecología en 1930, así como a Wolfang Tischler quien posiblemente realizó la primera publicación titulada Agroecología.

Hay más autores que se mencionan para el siglo $\mathrm{xx}$, pero no hay que dejar de lado los aportes de Stephen Gliessman desde Estados Unidos, Efraím Hernández Xolocotzi desde México, así como de Manuel Amador, Robert Netting y el mismo Miguel Altieri. En este recorrido se observan diversos intereses de estudio hasta la actualidad, donde a los agroecólogos les interesa principalmente “(...) analizar con cuidado el sistema alimentario global actual y a explorar alternativas locales en pro de formas de aprovisionamiento y acceso a los alimentos socialmente más justas y económicamente más viables" (Rosset y Altieri, 2019: p. 67).

En otras secciones del capítulo se discute cómo la antropología, etnoecología, sociología rural, entre otras disciplinas, han impactado en los estudios de agroecología. Este impacto se encuentra principalmente en la discusión del desarrollo rural y en colocar en el centro de sus estudios a las personas que poseen los sistemas agrícolas y que los han desarrollado desde muchos años atrás. Además, se encuentra un resumen del debate entre los descampesinistas y los campesinistas. Si bien este resumen es apropiado en extensión de cuartillas, se nota la ausencia de otros autores, por ejemplo: Ángel Palerm, Eric Wolf, Arturo Warman, entre otros.

Por último, el capítulo ofrece un breve panorama de otras corrientes de agricultura alternativa. Por citar, el papel de la agricultura orgánica, del comercio justo, y la postura de land saving (ahorro de tierras) frente a la de land sharing (tierra compartida). Ambas posturas pretenden conservar la biodiversidad, pero difieren en el tipo de agricultura que debe emplearse. También se describe la postura de la matriz de la naturaleza, que intenta compaginar conservación de biodiversidad, producción de alimentos y soberanía alimentaria. Por último, describen al ecofeminismo, 
una corriente dentro de la agroecología que visibiliza los aportes de la mujer campesina.

En el tercer capítulo, "Evidencias de la efectividad de la agroecología", los autores parten de la premisa sobre el hecho de que aumentar la producción de alimentos no reduce la pobreza si no se altera la concentración del poder económico. En este sentido, ellos muestran las evidencias de casos específicos en los cuales las prácticas y el manejo agroecológico permite incrementar la productividad de los agroecosistemas, contribuye al mejoramiento de la seguridad alimentaria y aumenta el ingreso económico de las familias campesinas. A lo largo del capítulo se utilizan ejemplos exitosos provenientes de África, Asia, Latinoamérica, Cuba, Chile y Brasil.

Algunas prácticas como el sistema push-pull para el manejo de plagas agroecológico, así como el sistema $Z a i$ basado en la cosecha de agua en la áfrica subsahariana o, el sistema de intensificación de arroz utilizado en China, Indonesia, Camboya y Vietnam sirven de evidencias de la efectividad de la agroecología. También se describen el movimiento "Campesino a Campesino" y otras estrategias agrícolas como la "Agricultura Natural de Presupuesto Cero". A partir de la ejemplificación de estos sistemas y estrategias, los autores señalan que la agricultura familiar puede ser más productiva y resiliente que una finca de monocultivo grande.

El cuarto capítulo, "Llevar a escala la agroecología", contiene un análisis de los procesos sociales que han permitido mantener vigente la agricultura agroecológica. Este capítulo afirma que existen muchos argumentos en favor de la transformación agroecológica de los sistemas agrícolas; se preguntan los autores cómo llevar a la agroecología a una escala superior. Para responder a esta interrogante hacen una revisión del concepto de escalamiento desde sus aristas cuantitativas, funcionales, políticas y de organización. Posteriormente, las reagrupan en un escalamiento vertical y horizontal. El primero se refiere a la institucionalización de las políticas de apoyo a la agricultura agroecológica.
El segundo, un escalamiento horizontal, implica que más familias y territorios lleven a cabo prácticas agroecológicas. De esta forma, vertical y horizontalmente se trata de masificar la agroecología, o de construir territorios agroecológicos. Sin embargo, existen barreras a romper, los autores encuentran ocho: 1) problemas de tenencia de tierra, 2) necesidades de los agricultores en materia de conocimiento y de información, 3) sesgos persistentes, barreras ideológicas y epistemológicas y falta de conocimientos prácticos, 4) especificidad de cada lugar, 5) falta de organizaciones campesinas, 6) barreras económicas, 7) políticas agrícolas nacionales, y 8) problemas de infraestructura. Cada obstáculo es bien detallado y ejemplificado.

El resto del capítulo detalla cómo la organización es fundamental para romper las barreras antes mencionadas, utilizando como ejemplos los movimientos "Campesino a Campesino", "La Vía Campesina", las escuelas de agroecología y la agricultura natural con presupuesto cero. De estos casos exitosos los autores extraen ocho factores que consideran reproducibles: 1) organización social, 2) metodología y pedagogía de procesos sociales horizontales, 3) protagonismo campesino, 4) prácticas agronómicas que funcionen, 5) discurso y marco motivadores, 6) oportunidad política, aliados externos, líderes carismáticos, campeones locales, 7) conexión de la producción local con los mercados locales y regionales, 8) políticas públicas favorables.

En el quinto y último capítulo, "La política de la agroecología", se encuentra un interesante debate sobre la agroecología como un terreno en disputa tanto en su arena material (agroecology as farming) como en su arena inmaterial (agroecology as framing). Tal como se planteó al inicio del libro, y de esta reseña, el capítulo está centrado en la parte política que es inherente a la agroecología. Los autores detallan cómo el sistema económico dominante intenta apropiarse de la agroecología para despojarla de su contenido, de sus principios y sus pilares ideológicos y utilizarla para "maquillar de verde" la agricultura convencional. 
A lo largo del capítulo se da un seguimiento cronológico a la forma en que la FAO abandera el movimiento agroecológico y las vicisitudes que ha enfrentado en esta lucha. Por ejemplo, los obstáculos de países como Estados Unidos que rechazan la agroecología o, en todo caso, intenta concebirla solamente como una simple técnica de producción orgánica en monocultivos, en lugar de respetarla como ciencia popular, surgida de los pueblos y que preconiza la justicia distributiva y pugna por una transformación del sistema alimentario. Sin discusión, este capítulo es la cereza del pastel.

Sin lugar a dudas, es un libro que nos hace reflexionar sobre lo que consumimos y sobre la forma en que es producido. También es un libro que nos puede inspirar hacia el desarrollo de determinadas investigaciones y resolución de problemas científicos. Además, puede servir de base para comprender el estatus en el que se encuentran nuestros agricultores y campesinos, que día a día trabajan en sus pequeñas fincas familiares y abastecen de comida a una gran parte del mundo. Ojalá esta breve reseńa sirva para que la comunidad científica se interese por el libro y por la agroecología.

Rosset, P., y Altieri, M. (2019). Agroecología: ciencia y política. Ciudad de México, México: Universidad Autónoma de Zacatecas, Miguel Ángel Porrúa. 